\title{
Noncontact magnetically controlled capsule endoscopy for infection-free gastric examination during the COVID-19 pandemic: a pilot, open-label, randomized trial
}

다(1)우우

\author{
Authors \\ Linghu' ${ }^{2}$, Zhao-Shen Li ${ }^{1}$, Zhuan Liao ${ }^{1}$ \\ Institutions \\ 1 National Clinical Research Center for Digestive Diseases, \\ Department of Gastroenterology, Changhai Hospital, \\ Shanghai, China \\ 2 Department of Gastroenterology, The First Medical \\ Center of PLA General Hospital/Chinese PLA \\ Postgraduate Military Medical School, Beijing, China
}

Jia-Hui Zhu*, 1, Jun Pan*, 1, Xiao-Nan Xu*, 1, Ya-Wei Liu* ,2, Yang-Yang Qian ${ }^{1}$, Xi Jiang ${ }^{1}$, Bin Jiang ${ }^{1}$, Wei Zhou ${ }^{1}$, En-Qiang

submitted 3.3.2021

accepted after revision 17.8 .2021

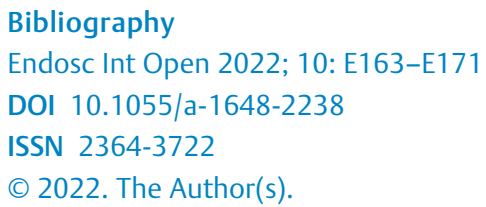
Commons Attribution-NonDerivative-NonCommercial License, permitting copying and reproduction so long as the original work is given appropriate credit. Contents may not be used for commercial purposes, or adapted, remixed, transformed or built upon. (https://creativecommons.org/licenses/by-nc-nd/4.0/)

Georg Thieme Verlag KG, Rüdigerstraße 14,

70469 Stuttgart, Germany

Corresponding author

Prof. Zhuan Liao, Department of Gastroenterology, Changhai Hospital, 168 Changhai Road, Shanghai 200433, China Fax: +86-21-55621735

liaozhuan@smmu.edu.cn

Supplementary material is available under https://doi.org/10.1055/a-1648-2238

\section{ABSTRACT}

Background and study aims Endoscopists have been at increased risk because of their direct contact with patients during the COVID-19 pandemic. For patients, being diagnosed with and monitored for gastrointestinal cancer and digestive diseases in timely fashion has been challenging, ments. We developed a novel noncontact magnetically controlled capsule endoscopy (ncMCE) system in our medical center. In the current study, we aimed to evaluate the feasibility and safety of ncMCE for gastric examination.

Patients and methods Patients were randomly assigned to groups that received ncMCE or MCE in a 1:1 ratio from March 26, 2020 to April 26, 2020. Primary endpoints were feasibility assessed by completion rate (CR) and safety based on the occurrence of adverse events (AEs) including infection. Secondary endpoints included maneuverability of endoscopists, pre-procedure perception and post-procedure satisfaction of patients, gastric examination time (GET), and diagnostic yield (DY).

Results Forty patients were enrolled with $100 \%$ CR in both groups without any AEs. Neither the endoscopist nor the patients were infected with severe acute respiratory syndrome coronavirus 2 (SARS-CoV-2) within 14 days after gastric examination. There were no significant differences in maneuverability ( 19.3 vs. $20.0, P=0.179$ ), pre-procedure perception ( 9 vs. $9, P=0.626$ ) and post-procedure satisfaction (45 vs. $44, P=0.999)$, ord DY ( $20 \%$ vs. $30 \%, P=0.465)$. Conclusions ncMCE is a feasible and safe method of gastric examination, which has the potential to protect both medical staff and patients from COVID-19 infection while providing serving as an essential endoscopy service. given pandemic-related adjustments in endoscopy depart-

\section{Introduction}

COVID-19, a respiratory disease that spreads via direct contact or through droplet transmission, has caused a major disruption

\footnotetext{
* These authors contributed equally
}

in the healthcare system since it was first identified in 2019 [1] Endoscopic procedures during the pandemic were divided into urgent endoscopy, semi-urgent endoscopy, and elective endoscopy, with the aim of preventing infection among healthcare professionals and providing necessary medical services [2]. For patients who required urgent gastroscopy, the face-to-face 
contact with endoscopists, aggressive suctioning, and coughing [3] during examination brought opportunities for transmission. Considering the reduced endoscopy volume in most endoscopy departments, diagnosis of patients who needed semi-urgent endoscopy and elective endoscopy may have been delayed [4]. Therefore, focusing on how to return to gradually return to provision of routine endoscopy services while maintaining safety for endoscopists deserves attention [5].

Capsule endoscopy provides a great view of the gastrointestinal tract through uyse of a miniature, pill-sized camera and it is as accurate as other gastrointestinal endoscopy modalities [6]. Its unique advantages include single use, excellent tolerance, and minimal medical staff requirement, which reduces the risk of exposure to pathogenic microorganisms and crossinfection [7] and makes it an appropriate method for examining and triaging an endoscopy department during the COVID-19 pandemic [8].

However, capsule endoscopy cannot be used to avoid faceto-face contact between endoscopists and patients who have asymptomatic COVID-19 cases [9]. Herein, we describe a novel noncontact magnetically controlled capsule endoscopy (ncMCE) system using a remote control system to separate endoscopists and patients physically, which was developed and tested in our medical center $[10,11]$. The objective of the present study was to evaluate the feasibility and safety of ncMCE for gastric examination.

\section{Patients and methods}

\section{Study design and patients}

This study was a pilot, open-label, randomized controlled trial approved by the institutional review board of Shanghai Changhai Hospital and registered at ClinicalTrials.gov (NCT 04389333). All of the diagnostic and therapeutic modalities were carried out in accordance with the approved guidelines.

Beginning on March 26, 2020, we consecutively recruited 40 patients in Changhai Hospital using predefined inclusion criteria and exclusion criteria. After fully understanding the procedure of ncMCE and this study, patients who gave informed consent were randomly assigned into ncMCE and MCE groups (in a $1: 1$ ratio) by using a stratified block randomization (fixed block size four). Randomization was based on a computer-generated list of random numbers using SPSS software (version 22.0, SPSS Inc., Chicago, Illinois, United States). Only after the enrolled participants completed all baseline assessments did the endoscopist inform them about their allocation. Eligible patients were adults ( $\geq 18$ years of age) undergoing outpatient MCE. Patients with any of the following conditions were excluded: (1) dysphagia or symptoms of gastric outlet obstruction, suspected or known intestinal stenosis, overt gastrointestinal bleeding, fistulas and strictures; (2) history of upper gastrointestinal surgery or suspected delayed gastric emptying; (3) poor general condition, asthma or claustrophobia; (4) implanted metallic devices such as pacemakers, defibrillators, artificial heart valves or joint prostheses; (5) pregnancy or mental illness; (6) current participation in another clinical study; or (7) difficulty communicating.

\section{Study intervention}

The MCE system (Ankon Technologies Co., Ltd. Shanghai, China) consists of a guidance C-arm magnet robot, a computer workstation with ESNavi software, endoscopic capsule, capsule locator, and a vest-like data recorder that can receive capsule signal from both sides [12]. The ncMCE system adds a remote control workstation and an audio-visual exchange system to the original well-established MCE system, which was developed and tested at our medical center [10]. To simplify the preparation process, we embedded the data recorder in the examination bed. The ncMCE system separates endoscopist and patients in two rooms (control room for endoscopist and examination room for patients), offering physical isolation for noninvasive gastric examination during the pandemic ( $\triangleright$ Fig. 1) [11].

Patients were required to report personal history and have chest computed tomography or nucleic acid testing to exclude COVID-19 infection within 3 days before their examination. Patients who are confirmed to have a COVID-19 infection and symptoms of pneumonia should be treated at special medical institutions first. Only when this population needs immediate endoscopy should those procedures be done in specific wards that have COVID-19 infection prevention protocols in place [13].

On arrival at the hospital in the morning after an overnight fast (> 8 hours), each patient took a $400-\mathrm{mg}$ simethicone suspension dissolved in $100 \mathrm{~mL}$ water 40 minutes before they ingested the capsule ingestion according to standardized gastric preparation [14]. Another 800 to $1000 \mathrm{~mL}$ of water could be taken 10 minutes before capsule ingestion to distend the stomach [12]. Then, a patient's hands were disinfected and they were given the data recorder after entering the examination room. Next, a patient laid down on the examination bed next to the computer workstation, assumed the supine or left-lateral decubitus position, and swallowed the capsule, which had already been activated. An endoscopist controlled capsule movement with the help of a magnet robot using two joysticks in front of the workstation or automatically using a default mode. To ensure that the gastric mucosa was completely visualized, the endoscopist directed a patient to change position to supine and right-lateral decubitus. In the ncMCE group, a patient and the endoscopist entered two rooms through different routes and gastric preparation was under the remote guidance [15]. The audio-visual exchange system provided a platform for the endoscopist to see and communicate with a patient during examination. The data recorder was embedded in the examination bed to avoid cross-infection of COVID-19 among patients and simplify the disinfection process.

Enhanced personal protective equipment (PPE) including an N95 mask, isolation gown with water resistance, head cover, eye protection, and face shield [11] should be worn by medical staff in the capsule endoscopy examination room when performing MCE. The steps for donning and doffing PPE were strictly followed, according to the standard process [16]. Medical masks were used as basic PPE for endoscopists in the ncMCE group (Supplementary material, Table 1) [17]. After each examination, the returned data recorder was disinfected using ul- 


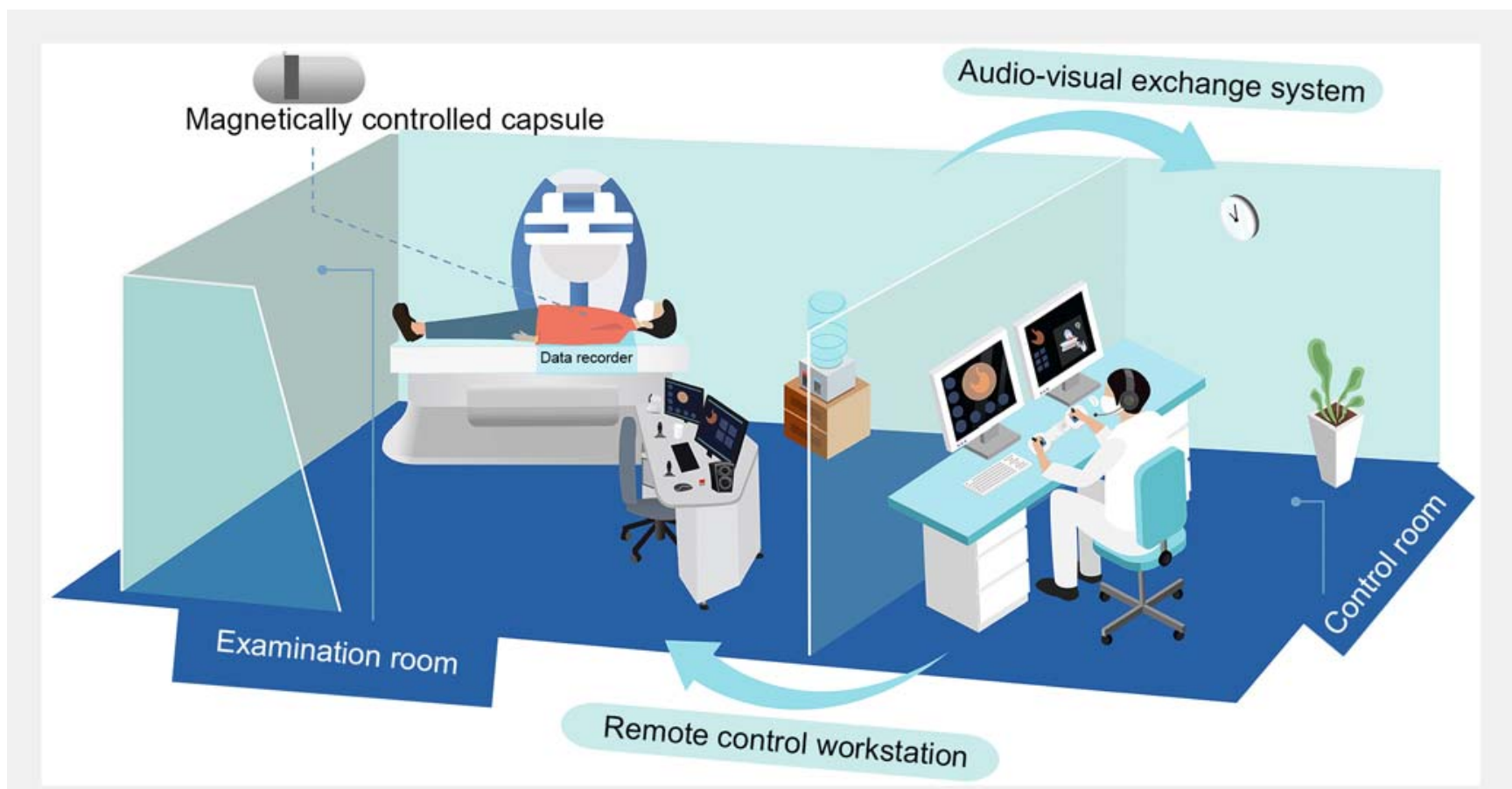

- Fig. 1 Schematic diagram of noncontact magnetically controlled capsule endoscopy Real-time communication via audio-visual exchange system and magnetic control precisely by a remote-controlled workstation ensured the safety of endoscopists and avoided the risk of cross-infection. Originally published in Liao Z, He S, Sha W, et al. Capsule endoscopy practice during the COVID-19 pandemic: Recommendations from the Capsule Endoscopy Group of the Chinese Society of Digestive Endoscopy. Endosc Int Open 2021; 9: E280-E283. Permission for reuse has been obtained from the authors.

traviolet light, $75 \%$ alcohol, or ethylene oxide and the sheets on the bed were replaced.

\section{Data collection}

Information on the basic characteristics of the enrolled patients was collected prospectively. Each patient was followed up for 2 weeks by telephone and hospital information system to confirm capsule excretion and any adverse events (AEs), including COVID-19 infection. The maneuverability questionnaire for the endoscopist and improved questionnaire on pre-procedure perception and post-procedure satisfaction for patients were completed after the examination. To avoid systematic bias, a single endoscopist (W.Z.) performed each examination and made the diagnoses for all enrolled patients immediately. The evaluation of completion was made by another blinded researcher (J.H.Z.).

\section{Study outcomes}

The primary endpoints were feasibility assessed by completion rate $(C R)$ and safety evaluated by the occurrence of $A E$. CR was defined as complete observation of the cardia, fundus, body, angulus, antrum, and pylorus of the stomach with no technical failure. Technical failure included incomplete observation, direct contact in the ncMCE group, and examination discontinuation. The safety of ncMCE was evaluated 2 weeks after the procedure for any AEs, such as infection with SARS-CoV-2 and capsule-related AEs including abdominal pain, nausea, vomiting, and capsule impaction or retention. In the current study, the endoscopist was continuously monitored for infection until 2 weeks after the last examination.

Secondary endpoints included maneuverability score, preprocedure perception and post-procedure satisfaction of patients, gastric examination time (GET), and diagnostic yield (DY). The maneuverability of the ncMCE system was evaluated with a questionnaire that consisted of signal transmission quality (stability and fluency), endoscopist comfort (strength needed to control joysticks and degree of fatigue), visualization of gastric mucosa (whether the mucus, foam in the stomach, and gastric fullness affected observation of gastric mucosa) and compliance of patients (patient ability to readily and correctly follow verbal instructions to change positions to assist in optimizing gastric views). The score for each index was 1 to 5 , with 1 as the worst and 5 as the best, respectively, and a total ranging from 4 to 20 . All patients were asked about the comfort and acceptability of the procedure using an improved pre-procedure perception and post-procedure satisfaction questionnaire, which was reviewed ease of swallowing, pain or discomfort experienced during and after the procedure, overall tolerability and convenience of the procedure, and knowledge, necessity for, and acceptability of ncMCE (Supplementary material, Questionnaire 1) [18]. GET was defined as the time taken for gastric examination and determined using a digital stopwatch in the ESNavi software. DY or detection rate was calculated using the following formula: number of patients with posi- 
- Table1 Baseline characteristic.

\begin{tabular}{|c|c|c|c|}
\hline & ncMCE group $(n=20)$ & MCE group $(n=20)$ & $P$ value \\
\hline Male & 11 & 8 & 0.342 \\
\hline Age, $y$, mean $\pm S D$ & $46.1 \pm 14.8$ & $53.5 \pm 9.2$ & 0.067 \\
\hline Body mass index, $\mathrm{kg} / \mathrm{m}^{2}$, mean $\pm \mathrm{SD}$ & $23.1 \pm 3.3$ & $23.5 \pm 3.2$ & 0.698 \\
\hline History of abdominal surgery & $4(20 \%)$ & $6(30 \%)$ & 0.465 \\
\hline Diabetes & $2(10 \%)$ & $1(5 \%)$ & 0.999 \\
\hline Smoking history & $3(15 \%)$ & $6(30 \%)$ & 0.449 \\
\hline Drinking history & $6(30 \%)$ & $7(35 \%)$ & 0.736 \\
\hline \multicolumn{4}{|l|}{ Indication } \\
\hline - Symptoms & $16(80 \%)$ & $14(70 \%)$ & 0.716 \\
\hline - Health checkup & $4(20 \%)$ & $6(30 \%)$ & 0.716 \\
\hline \multicolumn{4}{|l|}{ Indication } \\
\hline - Abdominal pain & $9(45 \%)$ & $3(15 \%)$ & 0.038 \\
\hline - Abdominal distension & $7(35 \%)$ & $6(30 \%)$ & 0.736 \\
\hline - Acid reflux & $0(0 \%)$ & $2(10 \%)$ & 0.487 \\
\hline - Health checkup & $4(20 \%)$ & $6(30 \%)$ & 0.465 \\
\hline - Others & $0(0 \%)$ & $3(15 \%)$ & 0.231 \\
\hline
\end{tabular}

tive findings divided by the total number of patients that underwent examination.

\section{Statistical analysis}

As a pilot study to evaluate the feasibility and safety of ncMCE, the sample size was not calculated [19]. Considering the capsule endoscopy volume of Changhai Hospital during the pandemic, we planned to recruit 40 patients into two groups. Basic characteristics were presented as means with standard deviations for continuous variables such as age, body mass index (BMI), and percentages for binary variables including sex, medical history, and symptoms. Quantitative data were summarized with parametric statistics, mean and standard deviation, or nonparametric statistics, median, and interquartile range (IQR), whereas categorical data were presented as frequency (percentage).

Under the condition of normal distribution and homogeneity of variance, variance analysis was used to compare the difference in mean between groups, while a K-W test was used to compare the difference in distribution between groups and Fisher's test was used to compare the two and disordered multi-classification data of subjects under the condition that homogeneity of variance was not satisfied. All reported $P$ values are two-sided and differences that reached $P<0.05$ were considered statistically significant. Statistical analyses were performed with SPSS (version 22.0, SPSS Inc., Chicago, Illinois, United States).

\section{Results}

Patients

From March 26, 2020 to April 26, 2020, 40 patients (21 men and 19 women) were enrolled and included in the analysis. Of them, 20 accepted MCE and 20 underwent ncMCE according to the random number table. The mean BMI of patients was $23.27 \mathrm{~kg} / \mathrm{m}^{2}$ and the indications in patients were counted (abdominal pain, 12 [30.0\%]; abdominal distension, 13 [32.5\%]; health checkup, 10 [25.0\%]; acid reflux, 2 [5.0\%]; and miscellaneous, $3[7.5 \%])$. Ten of 40 patients (25\%) had a history of surgery. Baseline patient characteristics are shown in $>$ Table 1.

\section{Feasibility and safety}

MCE and ncMCE were successfully performed on all patients with $100 \%$ CR in both groups. No infection and any suspicious symptoms in patients or the endoscopist or capsule-related AEs were reported during the 2-week follow-up period.

\section{Maneuverability score}

The median maneuverability score in the ncMCE group was 19.25 (IQR, 18.6-20.0), which was not significantly different than in the MCE group (median, 20.0; IQR, 19.0-20.0; $P=$ 0.179 ). The transmission quality in the ncMCE group (median, 4.5; range, 4.5-5.0) was significantly lower $(P<0.001)$ than in the MCE group (median 5.0; range, 5.0-5.0), which was accept- 
- Table2 Maneuverability of ncMCE and MCE.

\begin{tabular}{|c|c|c|c|}
\hline & ncMCE group $(n=20)$ & MCE group $(n=20)$ & $P$ value \\
\hline \multicolumn{4}{|l|}{ Maneuverability, median (IQR) } \\
\hline - Transmission quality & $4.5(4.5-5)$ & $5(5-5)$ & 0.001 \\
\hline - Comfortableness & $5(5-5)$ & $5(5-5)$ & 0.487 \\
\hline - Visualization & $5(5-5)$ & $5(4-5)$ & 0.716 \\
\hline - Compliance of patients & $5(5-5)$ & $5(5-5)$ & 0.999 \\
\hline - Total & $19.25(18.63-20)$ & $20(19-20)$ & 0.179 \\
\hline \multicolumn{4}{|c|}{$\begin{array}{l}\text { The signals transmission quality includes the stability and fluency of image and the comfortableness of endoscopist includes the strength to control joysticks an } \\
\text { fatigue degree, which are based on the score of MCE group is } 5 \text { (Supplementary material, questionnaire 2). } \\
\text { ncMCE, noncontact magnetically controlled capsule endoscopy; MCE, magnetically controlled capsule endoscopy; IQR, interquartile range. }\end{array}$} \\
\hline Item & ncMCE group $(n=20)$ & MCE group $(n=20)$ & $P$ value \\
\hline \multicolumn{4}{|l|}{$\begin{array}{l}\text { Pre-procedure patient } \\
\text { perception, median (IQR) }\end{array}$} \\
\hline - General anxiousness & $4(3-4)$ & $4(3-4)$ & 0.802 \\
\hline - Fear of pain & $4(4-4)$ & $4(4-4)$ & 0.553 \\
\hline - Understanding & $1(1.0-1.8)$ & $1(1-1)$ & 0.077 \\
\hline - Total & $9(8-9)$ & $9(8-9)$ & 0.626 \\
\hline \multicolumn{4}{|l|}{$\begin{array}{l}\text { Post-procedure patient } \\
\text { satisfaction, median (IQR) }\end{array}$} \\
\hline - Ease of swallowing & $4(4-4)$ & $4(4-4)$ & 0.595 \\
\hline - Pain during procedure & $4(4-4)$ & $4(4-4)$ & 0.152 \\
\hline - Discomfort during procedure & $4(4-4)$ & $4(4-4)$ & 0.152 \\
\hline - Pain after procedure & $4(4-4)$ & $4(4-4)$ & 0.317 \\
\hline - Discomfort after procedure & $4(4-4)$ & $4(4-4)$ & 0.152 \\
\hline - Comfort level & $4(3-4)$ & $3(3-4)$ & 0.294 \\
\hline - Selected procedure & $3(3-4)$ & $3.5(3-4)$ & 0.522 \\
\hline - Overall convenience & $4(3.3-4.0)$ & $3.5(3-4)$ & 0.107 \\
\hline - Missed time from work & $3.5(3-4)$ & $4(3-4)$ & 0.608 \\
\hline - Necessity & $3.5(3-4)$ & $3(2.0-3.8)$ & 0.121 \\
\hline - Inconvenience & $4(3-4)$ & $3.5(3-4)$ & 0.174 \\
\hline - Acceptance & $4(4-4)$ & $4(4-4)$ & 0.743 \\
\hline - Total & $45(42.3-46.8)$ & $44(42-45)$ & 0.999 \\
\hline
\end{tabular}

able for endoscopists. Whereas the comfortableness, visualization and compliance of patients were similar in both groups ( Table 2).

\section{Pre-procedure perception and post-procedure satisfaction}

There was no significant difference in pre-procedure perception ( 9 vs. $9, P=0.626$ ) or post-procedure satisfaction (45 vs.
$44, P=0.999$ ) in patients in the two groups ( $>$ Table 3 ). Time off work for all the patients was less than 6 hours as a result of ncMCE or MCE. Both ncMCE and MCE were acceptable to them (score for 15 th question $\geq 2$ ) with no inconvenience. Ninety-five percent of patients agreed with the necessity for ncMCE during the pandemic (score for 13th question $\geq 2$ ). 

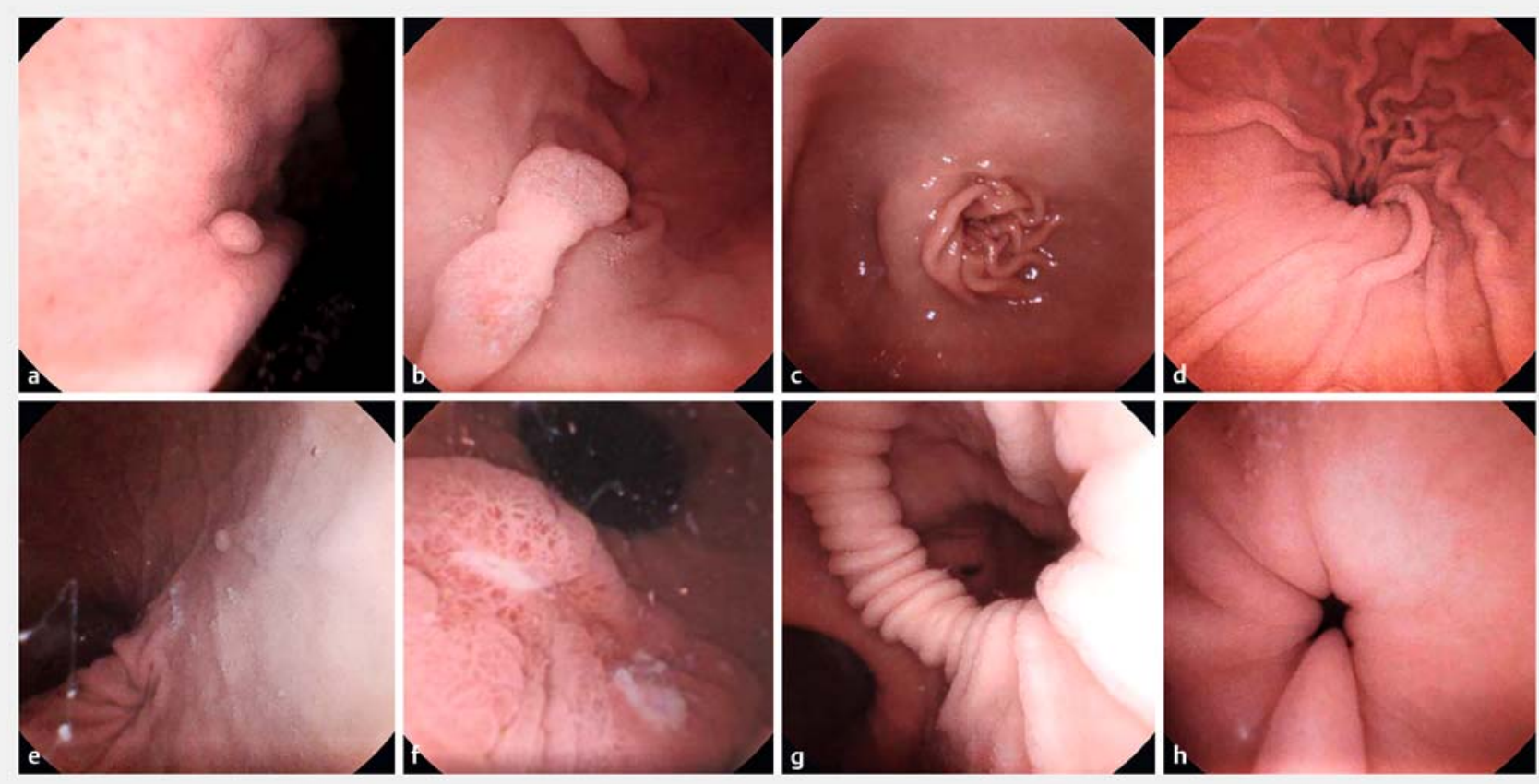

- Fig.2 Findings of noncontact magnetically controlled capsule endoscopy. Representative gastric diseases observed by ncMCE (upper panel) and MCE (lower panel). a Gastric polyp. b Antrum erosion. c Normal antrum. d Normal gastric body. e Gastric polyp. f Gastric ulcer. $\mathbf{g}$ Normal gastric angle. h Normal pylorus.

- Table 4 Diagnostic yield of ncMCE and MCE.

\begin{tabular}{|l|l|l|l|}
\hline & $\begin{array}{l}\text { ncMCE group } \\
(\mathbf{n = 2 0 )}\end{array}$ & $\begin{array}{c}\text { MCE group } \\
(\mathbf{n = 2 0 )}\end{array}$ & P value \\
\hline \multicolumn{2}{|c|}{ Diagnostic yield, $\mathrm{n}(\%)$} & \\
\hline - Polyp & $2(10 \%)$ & $3(15 \%)$ & \\
\hline - Erosion & $2(10 \%)$ & $1(5 \%)$ & \\
\hline - Ulcer & $0(0 \%)$ & $2(10 \%)$ & 0.465 \\
\hline Total & $4(20 \%)$ & $6(30 \%)$ & \\
\hline $\begin{array}{l}\text { ncMCE, noncontact magnetically controlled capsule endoscopy; MCE, mag- } \\
\text { netically controlled capsule endoscopy. }\end{array}$ & \\
\hline
\end{tabular}

\section{GET and diagnostic yield}

The GET in the ncMCE group was 12.3 minutes on average (IQR, 7.5-16.3), which was longer than the 7.2 minutes in the MCE group (IQR, 6.5-8.9; $P=0.003)$. There was no obvious difference between the two groups in positive findings $(P=0.465)$. Four patients (20\%) in the ncMCE group had positive findings as did six patients $(30 \%)$ in the MCE group ( $\downarrow$ Table 2 ). Two (10\%) and three (15\%) patients were diagnosed with polyps and erosion was detected in two (10\%) and one (5\%) patient in the ncMCE and MCE groups, respectively. The other two patients in the MCE group were diagnosed with ulcer ( $\mathbf{F i g . 2 ,}$ - Table 4).

\section{Discussion}

To the best of our knowledge, this study is the first randomized trial to evaluate the feasibility and safety of ncMCE compared with MCE during the COVID-19 pandemic. We observed that nCMCE, which had $100 \%$ CR and safety with excellent acceptance and tolerance, was as maneuverable as MCE and offered physical isolation.

Since the COVID-19 outbreak, a series of infection prevention measures and guidelines have been published for endoscopy departments [20]. ncMCE is a feasible method for endoscopists and patients, especially for high-risk gastric examination during the COVID-19 pandemic.

For endoscopists working in critical and uncertain conditions of the pandemic, ncMCE may protect medical staff from infection with SARS-CoV-2 by ensuring physical isolation. Medical staff are facing great challenges to their physical and mental health. In one of the earliest documented cases of infection in Wuhan, 29\% of patients were healthcare workers [21]. A $13.7 \%$ prevalence of SARS-CoV-2 antibodies was reported among health workers in a large cohort study in New York State [22]. These findings remind us that the risk of infection in health workers may higher than is expected. Standard or enhanced PPE should be used by personnel who are on the front lines against COVID-19. But the use of PPE leads to decreased work efficiency and medical resource shortages [23], which can be solved by ncMCE. In the current study, a questionnaire was used to innovatively quantify the device's maneuverability. We did not detect any significant difference in the score for maneuverability between the two groups, except for signal transmis- 
sion quality. The endoscopist may have had to talk to the patients in the ncMCE group more frequently, but it did not affect his or her comfortableness with the technology.

For patients who need gastric examination, ncMCE offers an infection-free method to avoid delay in diagnosis and monitoring. A study has shown that the number of patients diagnosed with gastric cancer dropped by $49.1 \%$, and $4.6 \%$ of gastric cancer patients would have higher stage with a 6-month shift during the COVID-19 pandemic [24]. In our previous study, a cardia gastric cancer was found in 75-year-old man with ncMCE, which underscores the need for more suitable endoscopy during COVID-19 [10]. The use of ncMCE solved the conflict between medical requirement and personal protection, given the $100 \%$ CR with AEs in our study. Safety was associated with use of strict personal protection measures by the endoscopist for the MCE group and physical isolation in the ncMCE group. The $100 \%$ CR was due to the simple preparation and examination procedure, which could be understood and completed easily by patients through the remote guidance of the endoscopist. As a method of gastrointestinal examination, the diagnostic ability of ncMCE was similar to that for MCE. The standardized gastric preparation and proficient operation of the endoscopist led to good visualization of the gastric mucosa. Positive findings were present in one-quarter of all our patients totally, which was consist with the rate of findings in western and eastern countries [25].

Occasionally, the signal was delayed during the examination procedure, which led to lower transmission quality and longer GET in the ncMCE group. The data recorder in the ncMCE group was put on the examination bed instead of being worn on the body, so the signal receiving mode changed from bilateral to dorsal. When patients turned to the right-lateral decubitus position, the antrum was the highest point in the whole stomach and the farthest from the data recorder, which theoretically may lead to longer signal transmission time. In addition, the extra time spent on communication with patients and instructing them to change their positions also contributed to the longer GET. However, the time to wear PPE for endoscopists and wear the data recorder for patients can be saved in the ncMCE group.

Our study is novel in three ways. First, it was an exploration of infection-free endoscopy for the first time, which is necessary not only during COVID-19, but also in special cases, such as patients who have influenza, radiation injury, or impaired immunity. Second, ncMCE provide a new method of using telemedicine in endoscopy. Telemedicine or virtual visits is a part of the emergency response in some countries [23] because of the better view, higher precision of an operation [26], fewer outpatient visits [27], and complete isolation between patients and doctors [28]. The technology is developing rapidly and likely will remain in place after the pandemic [29]. As with the DaVinci robotic system for surgery [26], in the field of remote manipulation endoscopy, a robot-assisted system named YunSRobot was shown to result in no obvious differences in percentage of clear view time or longer inspection time, compared with traditional manual endoscopy [30]. Unlike other telemedicine devices, the ncMCE system can be built easily in a short time ( $<10$ minutes) with no other costs except for one data line and two computers or even a glass partition if conditional, which means there was no extra financial burden on patients.

Third, we were particularly concerned about the maneuverability for endoscopists during the pandemic and designed a questionnaire to quantify that innovatively. According to previous studies, maneuverability is the first of three issues that need to be resolved for use of the stomach capsule, the other two being location and gastric preparation [31]. Maneuverability should be focused on during the pandemic because of the special working environment and personal protection requirements, which is different from the typical situation.

Some limitations of our study should be acknowledged. First, there no patients with confirmed SARS-CoV-2 infection were enrolled in this study because of the substantial reductions in COVID-19 transmission in China at that time [32], which led to the $0 \%$ infection rate in both groups. However, the technical feasibility (100\% CR) shown in our study suggests the potential for preventing COVID-19 infection, which needs confirmation in other centers. Second, the current study was single-center and had a small sample size with no blinding of patients, which may have caused subjective bias. Third, to limit subjective bias, we chose the same endoscopist for the two groups, which made the transmission of COVID-19 between patients and endoscopist untraceable. Furthermore, as a kind of capsule endoscopy, ncMCE also had a limitation in terms of taking biopsies and therapeutic interventions compared to esophagogastroduodenoscopy (EGD) [33].

With the development of wireless communication technology, remote surgery can be carried out, which requires high data and speed capacity and a long operation time over a reliable network with very low transmission delay [34]. With the help of wireless communication technology and an artificial intelligence-based auxiliary diagnostic system, a "one to many" medical model and high-quality community health care system will be built, and the shortage of endoscopists can be overcome. Considering the requirement for epidemic prevention and control worldwide for a long time, we believe that telemedicine is suitable for triaging patients before they arrive in the emergency department [35].

\section{Conclusions}

In conclusion, our novel ncMCE provides a feasible and safe method of gastric examination during the COVID-19 pandemic, which has the potential for preventing infection between endoscopists and patients and unintended harm from diagnostic delays to patients. It might be the first step in use of telemedicine for capsule endoscopy.

\section{Acknowledgements}

This study was sponsored by the Shanghai Municipal Hospital Emerging Frontier Technology Joint Project (to Z. Liao, No. SHDC12019105). 
Competing interests

The authors declare that they have no conflict of interest.

References

[1] Li Q, Guan X, Wu P et al. Early transmission dynamics in Wuhan, China, of novel coronavirus-infected pneumonia. N Engl J Med 2020; 382: 1199-1207

[2] Chiu PWY, Ng SC, Inoue $\mathrm{H}$ et al. Practice of endoscopy during COVID19 pandemic: position statements of the Asian Pacific Society for Digestive Endoscopy (APSDE-COVID statements). Gut 2020; 69: 991996

[3] Gu J, Han B, Wang J. COVID-19: Gastrointestinal manifestations and potential fecal-oral transmission. Gastroenterology 2020; 158: 15181519

[4] Lui TK, Leung K, Guo CG et al. Impacts of COVID-19 pandemic on gastrointestinal endoscopy volume and diagnosis of gastric and colorectal cancers: a population-based study. Gastroenterology 2020; 159: 1164-1166. e3

[5] Perisetti A, Gajendran M, Boregowda U et al. COVID-19 and gastrointestinal endoscopies: Current insights and emergent strategies. Dig Endosc 2020; 32: 715-722

[6] Liao Z, Hou X, Lin-Hu EQ et al. Accuracy of magnetically controlled capsule endoscopy, compared with conventional gastroscopy, in detection of gastric diseases. Clin Gastroenterol Hepatol 2016; 14: 1266-1273.e1261

[7] Enns RA, Hookey L, Armstrong D et al. Clinical practice guidelines for the use of video capsule endoscopy. Gastroenterology 2017; 152: 497-514

[8] MacLeod C, Wilson P, Watson AJM. Colon capsule endoscopy: an innovative method for detecting colorectal pathology during the COVID-19 pandemic? Colorectal Dis 2020; 22: 621-624

[9] Rothe C, Schunk M, Sothmann P et al. Transmission of 2019-nCoV infection from an asymptomatic contact in Germany. N Engl J Med 2020; 382: 970-971

[10] Pan J, Li Z, Liao Z. Noncontact endoscopy for infection-free gastric examination during the COVID-19 pandemic. VideoGIE 2020; 5: 402403.e401

[11] Liao Z, He S, Sha W et al. Capsule endoscopy practice during the COVID-19 pandemic: Recommendations from the Capsule Endoscopy Group of the Chinese Society of Digestive Endoscopy. Endosc Int Open 2021; 9: E280-E283

[12] Jiang X, Pan J, Li ZS et al. Standardized examination procedure of magnetically controlled capsule endoscopy. VideoGIE 2019; 4: 239243

[13] Chai N, Mei Z, Zhang W et al. Endoscopy works during the pandemic of coronavirus COVID-19: recommendations by the Chinese Society of Digestive Endoscopy. United European Gastroenterol J 2020; 8: 798-803

[14] Zhu SG, Qian YY, Tang XY et al. Gastric preparation for magnetically controlled capsule endoscopy: A prospective, randomized singleblinded controlled trial. Dig Liver Dis 2018; 50: 42-47

[15] Cortegoso Valdivia P, Le Grazie M, Gaiani F et al. Separated pathways in the endoscopy unit for COVID-19 patients. Gastrointest Endosc 2020; 92: 239
[16] Siddique SM, Sultan S, Lim JK et al. Spotlight: COVID-19 PPE and Endoscopy. Gastroenterology 2020; 159: 759

[17] Soetikno R, Teoh AYB, Kaltenbach T et al. Considerations in performing endoscopy during the COVID-19 pandemic. Gastrointest Endosc 2020; 92: 176-183

[18] de Franchis R, Eisen GM, Laine L et al. Esophageal capsule endoscopy for screening and surveillance of esophageal varices in patients with portal hypertension. Hepatology 2008; 47: 1595-1603

[19] Eldridge SM, Chan CL, Campbell MJ et al. CONSORT 2010 statement: extension to randomised pilot and feasibility trials. Pilot Feasibility Stud 2016; 2: 64

[20] Gralnek IM, Hassan C, Beilenhoff U et al. ESGE and ESGENA Position Statement on gastrointestinal endoscopy and the COVID-19 pandemic. Endoscopy 2020; 52: 483-490

[21] Wang D, Hu B, Hu C et al. Clinical characteristics of 138 hospitalized patients with 2019 novel coronavirus-infected pneumonia in Wuhan, China. JAMA 2020; 323: 1061-1069

[22] Moscola J, Sembajwe G, Jarrett M et al. Prevalence of SARS-CoV-2 antibodies in health care personnel in the New York City Area. JAMA 2020; 324: 893-895

[23] Thompson CC, Shen L, Lee LS. COVID-19 in endoscopy: Time to do more? Gastrointest Endosc 2020; 92: 435-439

[24] May A, Manner H, Schneider M et al. Prospective multicenter trial of capsule endoscopy in patients with chronic abdominal pain, diarrhea and other signs and symptoms (CEDAP-Plus Study). Endoscopy 2007; 39: 606-612

[25] Zagari RM, Eusebi LH, Rabitti S et al. Prevalence of upper gastrointestinal endoscopic findings in the community: A systematic review of studies in unselected samples of subjects. J Gastroenterol Hepatol 2016; 31: 1527-1538

[26] Senapati S, Advincula AP. Telemedicine and robotics: paving the way to the globalization of surgery. Int J Gynaecol Obstet 2005; 91: 210216

[27] de Jong MJ, van der Meulen-de Jong AE, Romberg-Camps MJ et al. Telemedicine for management of inflammatory bowel disease (mylBDcoach): a pragmatic, multicentre, randomised controlled trial. Lancet 2017; 390: 959-968

[28] Calton B, Abedini N, Fratkin M. Telemedicine in the time of coronavirus. J Pain Symptom Manage 2020; 60: e12-14

[29] Zweig S. Patient-doctor telemedicine: virtual care in the era of COVID-19 and beyond. Mo Med 2020; 117: 175-176

[30] Yan B, Liu H, Yang YS et al. The robot-assisted system YunSRobot for soft endoscopy: a trial of remote manipulation on simulation models. Zhonghua Nei Ke Za Zhi 2018; 57: 901-906

[31] Ching HL, Hale MF, McAlindon ME. Current and future role of magnetically assisted gastric capsule endoscopy in the upper gastrointestinal tract. Therap Adv Gastroenterol 2016; 9: 313-321

[32] Azman AS, Luquero FJ. From China: hope and lessons for COVID-19 control. Lancet Infect Dis 2020; 20: 756-757

[33] Swain P. At a watershed? Technical developments in wireless capsule endoscopy. J Dig Dis 2010; 11: 259-265

[34] Ullah H, Gopalakrishnan Nair N, Moore A et al. 5G Communication: An Overview of Vehicle-to-Everything, Drones, and Healthcare UseCases. IEEE Access 2019; 7: 37251-37268

[35] Hollander JE, Carr BG. Virtually perfect? Telemedicine for Covid-19. N Engl J Med 2020; 382: 1679-1681 
CORRECTION

Noncontact magnetically controlled capsule endoscopy for infection-free gastric examination during the COVID-19 pandemic: a pilot, open-label, randomized trial

Jia-Hui Zhu, Jun Pan, Xiao-Nan Xu et al.

Endoscopy International Open 2022; 10: E163-E170.

DOI: $10.1055 / a-1648-2238$

In the above-mentioned article the corresponding author was corrected to Zhuan Liao. His correspondence address is:

Prof. Zhuan Liao

Department of Gastroenterology

Changhai Hospital

168 Changhai Road

Shanghai 200433

China

Fax: +86-21-55621735

liaozhuan@smmu.edu.cn

This was corrected in the online version on 17.03.2022. 